\title{
Intermediate and high-risk prostate cancer methylation
} \section{analysis}

\author{
Anastasiya Andreevna Kobelyatskaya \\ Laboratory of Postgenomic Research \\ EIMB RAS \\ Moscow, Russia \\ kaa.chel@mail.ru \\ Kirill Mikhailovich Nyushko \\ Urological department \\ FSBI NMRRC \\ Moscow, Russia \\ kirandja@yandex.ru
}

\author{
Elena Anatolevna Pudova \\ Laboratory of Postgenomic Research \\ EIMB RAS \\ Moscow, Russia \\ pudova_elena@inbox.ru \\ Boris Yakovlevich Alekseev \\ Urological department \\ FSBI NMRRC \\ Moscow, Russia \\ mnioi@mail.ru
}

\author{
George Sergeevich Krasnov \\ Laboratory of Postgenomic Research \\ EIMB RAS \\ Moscow, Russia \\ gskrasnov@mail.ru \\ Anna Victorovna Kudryavtseva \\ Laboratory of Postgenomic Research \\ EIMB RAS \\ Moscow, Russia \\ rhizamoeba@mail.ru
}

\begin{abstract}
Prostate cancer is one of the most important socially significant oncological diseases in men. To select an effective approach to therapy, prostate cancer is stratified into appropriate risk groups based on criteria such as TNM status, Gleason score and the level of prostate-specific antigen (PSA). However, to optimize therapy, additional informative markers are necessary, and the aim of this study is to search for these markers at the level of genome methylation. This work included methylation data of prostate cancer from The Cancer Genome Atlas project. The cohort involved patients with high (23 cases) and intermediate (103 cases) risk. As a result, 1,056 differentially methylated CpG sites were found between high and medium risk groups. CpG most interested sites: $\operatorname{cg} 17687367$ (chr13: 79936801), cg26874611 (chr5: 168147884), cg06989693 (chr5: 41409252), cg02226810 (chr6: 1605117), cg07736716 (regulation regions: 85 ): 85 to 91 (85): 85 858: 858: 858: 85: 86: 85: 86: 85: 86: 85: 86: 85: 86: 85: 85 .
\end{abstract}

Keywords - risk group, prostate cancer, methylation, TCGA

\section{Introduction}

Prostate cancer (PC) is one of the most important socially significant oncological diseases in men.. When disease is detected the question arises as to the choice of treatment tactics [1]. Treatment may consist of surgery, hormone therapy, or both. As a rule, determination of risk group is based on criteria such as TNM parameters, Gleason score, PSA level [2, 3, 4]. Considering above criteria and risk group a therapeutic concept is chosen. However, in practice these criteria are not enough to choose a therapeutic concept [2]. And patients receive either excessive or insufficient therapy. Additional markers of diagnosis are needed to optimize the choice of therapy. The aim of research is to identification differentially methylated $\mathrm{CpG}$ sites as possible diagnosis markers PC.

\section{Methods}

This work included PC samples methylation data of The Cancer Genome Atlas (TCGA) project. Either high (23 cases) or intermediate (103 cases) risk PC cases are involved into the cohort. Risk groups were determinate based on TNM parameters, Gleason score and PSA level. Differential methylation analysis was performed in statistical environment
R using EdgeR and BiSeq packages. The Mann-Whitney test, beta-regression and logistic regression models were used for statistical analysis.

\section{Results}

In this study we found out 1056 differentially methylated $\mathrm{CpG}$ sites between high and intermediate risk groups. From those we selected the most interested $\mathrm{CpG}$ sites that are in regulatory regions: cg17687367 (chr13: 79936801), cg26874611 (chr5: 168147884), cg06989693 (chr5: 41409252), cg02226810 (chr6: 1605117), cg07736716 (chr8: 85379411). Above $\mathrm{CpG}$ sites are hypermethylated in intermediate risk group cases. These hypermethylated $\mathrm{CpG}$ are covered by regulatory regions such as ENSR00000064172 (promoter flank), ENSR00000776896 (enhancer), ENSR00000753523 (promoter flank), ENSR00000191969 (promoter), ENSR00000332061 (transcription factor binding site), ENSR00000860893 (transcription factor binding site). The results will be used in further validation study.

\section{ACKNOWLEDGMENT}

This work was financially supported by the RFBR, grant no. 17-29-06083. This work was performed using the equipment of EIMB RAS "Genome" center (http://www.eimb.ru/ru1/ckp/ccu_genome_c.php).

\section{REFERENCES}

[1] Chang A.J., Autio K.A., Roach M., Scher H.I. (2014) High-risk prostate cancer-classification and therapy. Nat Rev Clin Oncol. 11(6):308-23. doi: 10.1038/nrclinonc.2014.68.

[2] Gerhauser C., Favero F., Risch T., Simon R., Feuerbach L., Assenov Y., et al. (2018) Molecular Evolution of Early-Onset Prostate Cancer Identifies Molecular Risk Markers and Clinical Trajectories. Cancer Cell. 10;34(6):996-1011.e8. doi: 10.1016/j.ccell.2018.10.016.

[3] Hurwitz L.M., Cullen J., Kim D.J., Elsamanoudi S., Hudak J., Colston M., et al. (2017) Longitudinal regret after treatment for low- and intermediate-risk prostate cancer. Cancer. 1;123(21):4252-4258. doi: 10.1002/cncr.30841.

[4] Serrano N.A., Anscher M.S. (2016) Favorable vs Unfavorable Intermediate-Risk Prostate Cancer: A Review of the New Classification System and Its Impact on Treatment Recommendations. Oncology (Williston Park). 30(3):229-36. 